\title{
Sphenopalatine Ganglion Stimulation in Neurovascular Headaches
}

\author{
Jean Schoenen \\ Headache Research Unit, Department of Neurology, CHR Citadelle, Liège University, Liège, Belgium
}

\section{Abstract}

The interest for the sphenopalatine ganglion (SPG) in neurovascular headaches dates back to 1908 when Sluder presented his work on the role of the SPG in 'nasal headaches', which are now part of the trigeminal autonomic cephalalgias and cluster headache (ICHD-III-beta). Since then various interventions with blocking or lesional properties have targeted the SPG (transnasal injection of lidocaine and other agents, alcohol or steroid injections, radiofrequency lesions, or even ganglionectomy); success rates vary, but benefit is usually transient. Here we briefly review some anatomophysiological characteristics of the SPG and hypotheses about its pathophysiological role in neurovascular headaches before describing recent therapeutic results obtained with electrical stimulation of the SPG. Based on results of a prospective randomized controlled study, SPG stimulation appears to be an effective treatment option for patients with chronic cluster headaches; efficacy data indicate that acute electrical stimulation of the SPG provides significant attack pain relief and in many cases pain freedom compared to sham stimulation. Moreover, in some patients SPG stimulation has been associated with a significant and clinically meaningful reduction in cluster headache attack frequency; this preventive effect of SPG stimulation warrants further investigation. For migraine attacks, the outcome of a proof-of-concept study using a temporary electrode implanted in the pterygopalatine fossa was less encouraging; however, an ongoing multicenter trial is evaluating the efficacy of long-term SPG stimulation against sham stimulation for acute and preventive treatment in patients with frequent migraine.

(c) 2015 S. Karger AG, Basel

Interest in the sphenopalatine ganglion (SPG) in neurovascular headaches dates back to 1908 when Sluder [1] presented his work on the 'role of the sphenopalatine (or Meckel's) ganglion in nasal headaches', which are now part of the trigeminal autonomic cephalalgias and cluster headache (CH) (ICHD-III-beta) [2]. Since then various interventions with blocking or lesional properties have targeted the SPG such as transnasal injection of lidocaine and other agents $[3,4]$, alcohol or steroid injections 
[5], radiofrequency lesions [6-8], or even ganglionectomy [9]. Success rates vary, but benefit is usually transient [10].

We will briefly review some anatomophysiological characteristics of the SPG and hypotheses about its pathophysiological role in neurovascular headaches before describing recent therapeutic results obtained with electrical stimulation of the SPG.

\section{Anatomophysiology of the Sphenopalatine Ganglion}

The SPG is the largest extracranial parasympathetic ganglion located in the pterygopalatine fossa, where it is closely associated with sensory and sympathetic fibers (see review by Khan et al. [11]). Its parasympathetic fibers originate in the superior salivary nucleus (SSN) of the pons and supply extra- and intracerebral arteries, the lacrimal gland, nasal mucosa, and conjunctiva. Sympathetic fibers come from the pericarotid plexus and pass through the SPG to innervate the lacrimal gland and nasal and palatine mucosa. Only a few fibers from the sphenopalatine branches of the maxillary nerve enter the SPG, forming the sensory root. They innervate areas such as the posterior nasopharynx via the lesser palatine nerve.

Physiologically, low-frequency electrical stimulations of the SPG $(10-20 \mathrm{~Hz})$ in animal studies produce dilatation of intra- and extracranial arteries, increased cerebral blood flow and plasma protein extravasation in dura mater.

The trigeminoparasympathetic reflex consists of a brainstem connection between trigeminal afferents and parasympathetic efferents of the facial nerve that synapse in the SPG. The reflex was broadly demonstrated in animal and human studies, showing that trigeminal ganglion stimulation leads to intra- and extracranial vasodilatation, increased regional cerebral blood flow and increased facial temperature, a response that is abolished by lesioning the facial nerve. Thus, when the trigeminal-parasympathetic reflex is activated, the postganglionic parasympathetic fibers from the SPG release neurotransmitters and vasodilators that activate sensory trigeminal fibers causing further activation of the trigeminal pain pathway, which in turn causes further parasympathetic outflow $[12,13]$ (fig. 1).

\section{Pathogenic Role of the Sphenopalatine Ganglion in Neurovascular Headaches}

There is no doubt that the cranial autonomic symptoms that characterize $\mathrm{CH}$ attacks are predominantly due to activation of the parasympathetic system, and thus the SPG. It is postulated that upon nociceptive activation of nociceptors belonging to the ophthalmic nerve during a $\mathrm{CH}$ attack, the trigeminoparasympathetic reflex is activated. Fibers from the trigeminocervical complex project to the superior salivatory nucleus and activate facial parasympathetic efferents. As parasympathetic activation leads to vasodilation of meningeal arteries, a vicious circle further increasing trigeminal 
Fig. 1. Schematic outline of the trigeminoparasympathetic circuit. Trigeminovascular afferents (dark lines) belonging to the visceral part of the ophthalmic nerve innervate the meninges and relay in the spinal trigeminal nucleus, from where the SSN can be activated via nucleus tractus solitarius. The parasympathetic fibers (gray dashed lines) innervate meningeal vessels, lacrimal glands, and nasal mucosa via the SPG, producing cranial autonomic symptoms on the side of the headache. The SSN can activate trigeminovascular nociceptors in the spinal trigeminal nucleus and can be activated by descending afferents, e.g. from the hypothalamus.

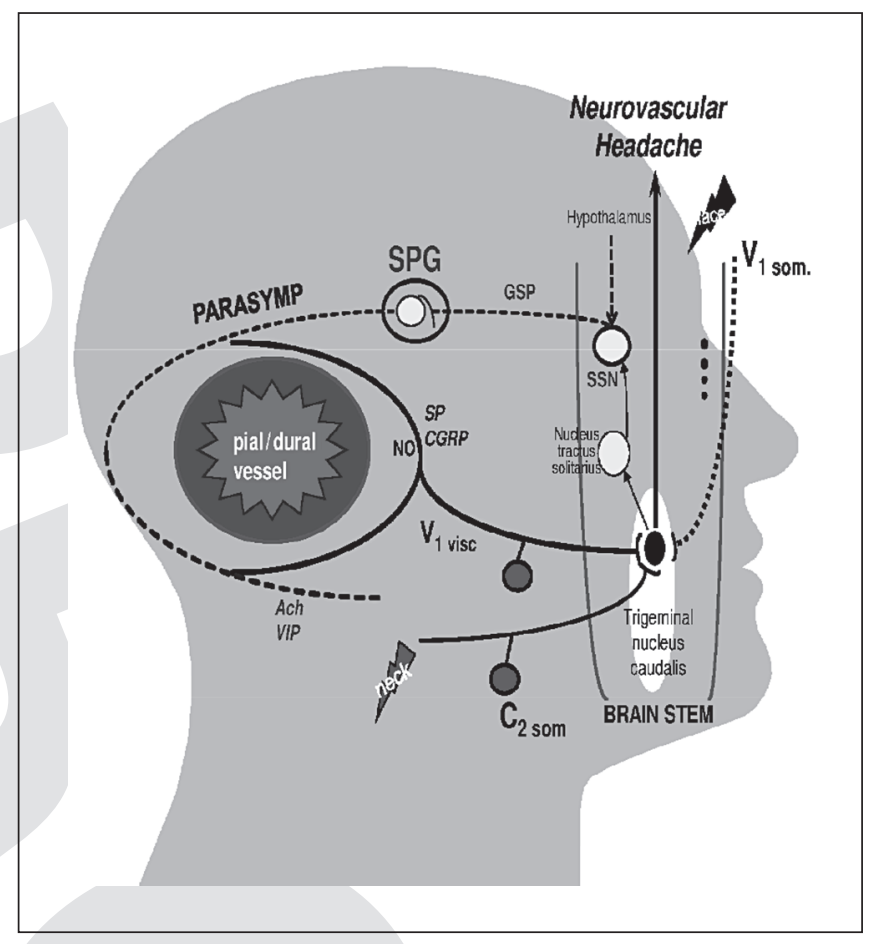

activation has been proposed [14]. This hypothesis is supported by a recent study showing that low-frequency stimulation of the SPG is able to induce attacks in $\mathrm{CH}$ patients [15]. Interestingly, low-frequency stimulation of the SSN in cats leads to firing of trigeminovascular neurons in the trigeminocervical complex, which is inhibited by oxygen inhalation, an established treatment for $\mathrm{CH}$ attacks [16].

Cranial autonomic symptoms may also occur during migraine attacks, although they are usually less prominent than in $\mathrm{CH}$. As mentioned, SPG activation produces meningeal vasodilatation and plasma protein extravasation, both of which may be involved in migraine pathogenesis [11]. Burstein and Jakubowski [17] have proposed that common migraine triggers, such as stress, may activate brain areas that project to the SSN. The SSN would then stimulate the release of vasoactive intestinal peptide, nitric oxide, and acetylcholine from meningeal terminals of SPG neurons. The resulting dilatation of intracranial blood vessel and local release of inflammatory molecules would activate meningeal nociceptors, and thus produce the migraine headache. The results of a randomized controlled trial (RCT) showing that during a migraine attack intranasal lidocaine $4 \%$ is able to relieve the headache in $35.8 \%$ of patients [18] could be considered as support for the 'parasympathetic' theory of migraine pathogenesis. However, there is no proof that lidocaine acts on the headache only via the SPG, and not by influencing sensory afferents. All things considered, the experimental evidence that the trigeminoparasympathetic circuit is pathogenically relevant is much weaker in migraine than in $\mathrm{CH}$. 
The SPG is thought to play a role in other pain syndromes besides headaches like trigeminal and sphenopalatine neuralgia, atypical facial pain, muscle pain, vasomotor rhinitis, eye disorders, and herpes infection. Clinical trials have shown that SPG blockade can be helpful in these pain disorders [19].

\section{Sphenopalatine Ganglion Neurostimulation: Available Data}

The data showing that blocking or lesioning the SPG may be helpful in neurovascular headaches and that low-frequency stimulation of the SPG in animals induces changes that may be associated with migraine and $\mathrm{CH}$ attacks led to the concept that highfrequency stimulation might on the contrary have an inhibitory effect that would be beneficial for acute treatment [11].

\section{Cluster Headache}

It is commonly accepted that subcutaneous injections of the 5-HT1B/D agonist sumatriptan are at present the most effective treatment for $\mathrm{CH}$ attacks. In the sole available RCT [20], pain relief and pain freedom at 15 min was obtained in 74 and $46 \%$ of patients, respectively. Sumatriptan, however, is contraindicated in patients with cardiovascular disease, and many $\mathrm{CH}$ patients are heavy smokers and thus at risk for cardiovascular disease. The next most effective acute therapy after sumatriptan is oxygen inhalation, which has a $78 \%$ success rate [21]. Various preventive treatments are available for $\mathrm{CH}$ such as steroids, verapamil, lithium, topiramate, methysergide, and valproate, but their use may be limited by intolerance or contraindications [22]. Moreover, $10-20 \%$ of patients develop the chronic form of $\mathrm{CH}$ without remissions and many of them become resistant to preventive drugs. In such patients, invasive neurostimulation methods were studied and found useful in $60-70 \%$ of the patients. Deep hypothalamic stimulation is effective but risky. Occipital nerve stimulation is as effective, but also associated with a rather high incidence of adverse effects, e.g. lead migration, infection, and battery depletion (for a review, see Magis and Schoenen [23]). Therefore, there is room in CH for safer acute treatments and for more effective and better tolerable preventive therapies.

In 2010, a proof-of-concept study on the response of $\mathrm{CH}$ patients to acute electrical stimulation of the SPG was published by Ansarinia et al. [24]. In 6 patients in whom attacks occurred spontaneously or were induced in the hospital, stimulation of the SPG with a removable stimulating electrode introduced in the pterygopalatine fossa abolished pain in 11 of 18 attacks (61\%) and reduced pain by $\geq 50 \%$ in 3 more attacks. Based on these data, Autonomic Technologies Inc. (ATI; Redwood, Calif., USA) developed an implantable SPG microstimulator and sponsored a prospective, randomized, blinded, multicenter study (Pathway $\mathrm{CH}-\mathrm{I}$ ) to test its efficacy and safety in drug-resistant chronic $\mathrm{CH}$ patients [25]. 


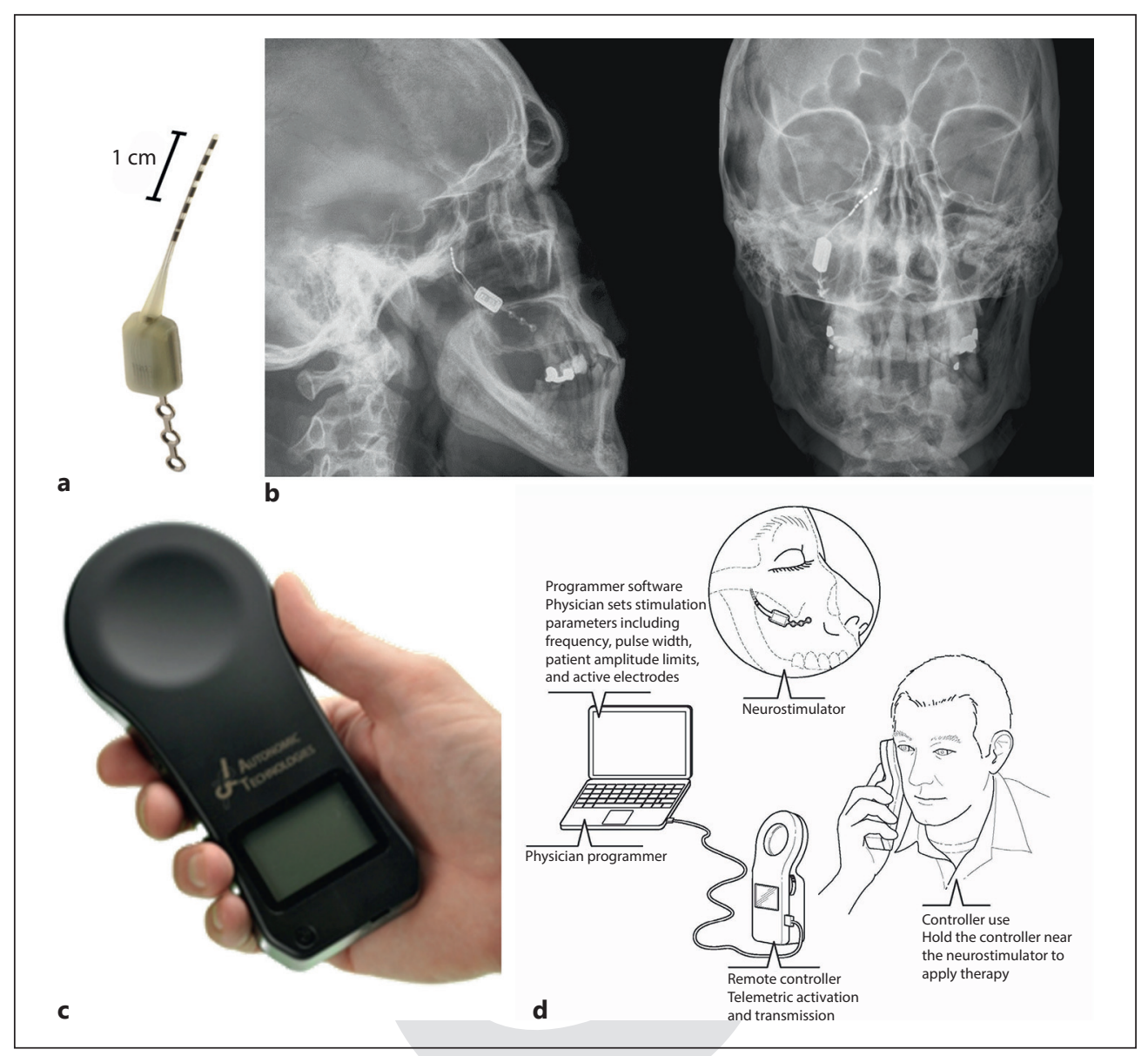

Fig. 2. The ATI Neurostimulator Pulsante ${ }^{T M}$ (a, available in four lengths) is a miniaturized device with an integral lead containing six electrodes that is implanted in the pterygopalatine fossa through the gums of the upper maxilla. $\mathbf{b}$ Lateral and anteroposterior X-rays showing the neurostimulator body anchored to the zygomatic process of the maxilla and the lead with its tip next to the anatomical location of the sphenopalatine ganglion. The handheld ATI Remote Controller (c) is positioned by the patient on the jaw to activate the implanted neurostimulator; it also allows the physician to program the stimulation parameters (d) (images courtesy of Autonomic Technologies Inc.).

\section{Protocol}

The ATI SPG Neurostimulator is implanted under general anesthesia using a minimally invasive, transoral, gingival buccal technique such that the stimulating electrodes on the integral lead are positioned within the pterygopalatine fossa close to the SPG and the body - on the lateral-posterior maxilla medial to the zygoma and anchored to the zygomatic process of the maxilla (fig. 2). The position of the SPG Neurostimulator is verified with an X-ray immediately after implantation, and, if needed, at later time points. Thirty-two chronic $\mathrm{CH}$ patients with unilateral attacks 
in 6 European clinical sites participated in the Pathway CH-I study. After postimplantation healing and titration periods, 4 patients had dropped out and 28 with a mean weekly attack frequency of 17.4 were randomized 1:1:1 to effective, subthreshold, or sham stimulation during an 8 -week or 30 -attack experimental period. Parameters of effective stimuli were adjusted to provoke paresthesias in the root of the nose or a treatment during an attack (mean intensity: $1.6 \mathrm{~mA}$; mean frequency: $120.4 \mathrm{~Hz}$; mean pulse width: $389.7 \mu \mathrm{s}$ ) and subperception stimuli were at $85 \%$ of the lowest effective amplitude. Patients were instructed to apply the remote controller on the face to treat ipsilateral $\mathrm{CH}$ attacks that were at least of moderate pain intensity (categorical pain scale: $0=$ none; $1=$ mild; $2=$ moderate; $3=$ severe; $4=$ very severe) and to stimulate for $15 \mathrm{~min}$, whereafter they were allowed to use acute medications if needed. Pain scores were recorded using a custom electronic headache diary included in the ATI Remote Controller prior to each use and 15, 30, 60, and 90 min after the start of stimulation. The primary efficacy endpoint was pain relief, i.e. a change from 2,3 , or 4 on the categorical pain scale to 0 or 1 at $15 \mathrm{~min}$ after the start of stimulation. Secondary endpoints were pain freedom at $15 \mathrm{~min}$, pain relief and freedom at 30,60, and $90 \mathrm{~min}$, as well as disability changes assessed by the Headache Impact Test (HIT-6) and quality of life changes assessed with the ShortForm Health Survey (SF-36v2). Safety was assessed by tabulating all device- or procedure-related serious adverse events from the implantation procedure to the end of the experimental period.

\section{Efficacy Data}

During the course of study, the SPG stimulation decreased attack frequency in a number of patients so that out of the 28 evaluable patients only 14 treated enough attacks to be analyzed for acute response. Pain relief at $15 \mathrm{~min}$ was achieved in $67.1 \%$ of attacks treated by these 14 patients with full stimulation, compared to $7.4 \%$ after sham stimulation and $7.3 \%$ after subthreshold stimulation. Pain freedom was obtained in 34.1 and $1.5 \%$, respectively. Among the 14 patients, $9(64 \%)$ were considered acute responders since they had pain relief at $15 \mathrm{~min}$ in at least $50 \%$ of the full stimulationtreated attacks. Overall, 9 out of the 28 patients in the experimental period (32\%) reported pain relief of $50 \%$ or more at 15 -min stimulation.

Twelve out of the randomized 28 patients (43\%) were frequency responders with an average $88 \%$ reduction of weekly attack frequency. In total, 19 patients out of 28 $(68 \%)$ experienced an acute response, a frequency response, or both: $\mathrm{n}=7(25 \%), \mathrm{n}=$ $10(36 \%)$, and $\mathrm{n}=2(7 \%)$, respectively. SPG stimulation resulted in a statistically and clinically significant reduction in headache disability.

On completion of the Pathway CH-I trial, patients had the opportunity to enroll in an open long-term follow-up. Among 14 patients who reached 18 months postimplantation, SPG stimulation (average duration $8.6 \mathrm{~min}$ ) was effective in $84 \%$ of the 
2,166 analyzable attacks. Efficacy was highest (93\%) in attacks of moderate severity, which were also the most frequent attacks treated with SPG stimulation (72\%), and lowest in very severe attacks (29\%).

\section{Safety Data}

As other maxillofacial surgeries, implantation of the ATI Neurostimulator induces standard perioperative adverse effects like local pain, swelling, hematoma, seroma, and sensory disturbances. Among the 32 operated patients in the Pathway CH-I trial, there were 5 device- or procedure-related serious adverse events: 3 misplacements needing a revision procedure, and 2 postimplantation migrations of the electrode needing explantation. Sensory disturbance in the distribution of the maxillary nerve occurred in $81 \%$ of the patients with loss of sensation being the most common (21 of 32 events in 19 of 32 patients). Sensory deficit resolved after an average of 97 days (range: 31-259), but in 6 patients, though being mild or moderate, it had not resolved at the time of the report, and in one patient with an electrode migration who had to be explanted because of neuropathic pain, a mild sensory deficit persisted 1 year after the procedure. During and after the Pathway $\mathrm{CH}$-I trial, advancements in surgical instruments and additional surgical experience have decreased surgical time by $25 \%$, the number of adverse events by $23 \%$, and the rate of explants or revisions by $83 \%$.

\section{Mechanism of Action}

As mentioned above, in a double-blind randomized crossover study, low-frequency $(5 \mathrm{~Hz})$ stimulation applied to the $6 \mathrm{CH}$ patients implanted with the ATI Neurostimulator triggered an attack in 3 of them. These attacks were effectively treated with highfrequency $(120 \mathrm{~Hz})$ stimulation [15].

The prominent autonomic symptoms associated with $\mathrm{CH}$ attacks result from increased cranial parasympathetic outflow that is thought to activate trigeminal afferents via the trigeminovascular system [12]. High-frequency electrical stimulation of the SPG may block the parasympathetic efferents, thereby turning off the efferent arm of the trigeminal-autonomic reflex, which is thought to be due to depletion of stored neurotransmitter(s). From a pathophysiological perspective, the positive results of the Pathway CH-I trial confirm that the cranial parasympathetic system plays a crucial role in the occurrence as well as recurrence of $\mathrm{CH}$ attacks.

Electrical stimulation in the region of the SPG also acts on the neighboring sensory fibers of the maxillary nerve, producing paresthesias in the nasopharynx, soft palate, nasal cavity, and palatal gingiva. In the Pathway CH-I study, these paresthesias guided the programming of stimulation parameters. Whether the activation of sensory fibers of the maxillary nerve contributes to the therapeutic effect is unlikely, but remains to be explored. 
The mechanisms of action may be different between the acute and preventive effects of SPG stimulation, which is suggested by the absence of a correlation between the two effects in many Pathway $\mathrm{CH}$-I patients. Assuming that it can be confirmed in an adequate RCT, the preventive effect might be associated with modulation of centers involved in descending pain control, similar to that found in chronic $\mathrm{CH}$ patients after occipital nerve stimulation [26].

\section{Migraine}

For the treatment of migraine attacks, analgesics, NSAIDs, and triptans are currently available. Triptans are the most effective, although the pain-free rate hardly exceeds $30 \%$ after $2 \mathrm{~h}$. Triptans may also have unpleasant side effects and they are contraindicated in patients with cardiovascular disease. Moreover, the overuse of analgesics and triptans is the most prevalent chronifying factor of migraine [27]. Preventive therapies in migraine have at best a $50 \%$ responder rate of $50-60 \%$, and the most efficient preventive drugs like the anticonvulsants topiramate and valproate are not tolerated by many patients. In addition, patients suffering from chronic migraine become notoriously resistant to preventive therapies [28]. Therefore, there is room for more efficacious and better tolerated treatments. In the last decade, several neuromodulation methods have been explored, including electrical stimulation of peripheral nervous structures [23]. Transcutaneous supraorbital nerve stimulation with a new device was found to be superior to placebo in an RCT of episodic migraineurs, but the $50 \%$ responder rate was just below $40 \%$ [29].

As mentioned above, there is some evidence for interictal autonomic dysfunctions in migraine, and cranial autonomic symptoms can accompany the attack [11]. It remains to be shown, however, whether these autonomic symptoms are epiphenomena due to the trigeminovascular activation or if they also contribute to the headache and central sensitization, as suggested by some trials [27].

A proof-of-concept study on the potential utility of SPG stimulation in migraine was conducted similarly to that performed in $\mathrm{CH}[30]$. As for $\mathrm{CH}$, the authors implanted a removable stimulation needle lead in the pterygopalatine fossa in 11 patients with medically refractory migraine: 9 with medication overuse headache and 2 with episodic migraine. The interventions were performed in an outpatient procedure room. Patients had spontaneous or induced migraine headaches and were, in a crossover protocol, either effectively or sham stimulated at the peak of their headache and associated migrainous features, i.e. several hours after pain onset. Patients had 1-3 peaks of headache stimulated for a mean of 3 min with a mean amplitude of $1.2 \mathrm{~V}$, mean frequency of $57 \mathrm{~Hz}$, and mean pulse width of $394 \mu \mathrm{s}$.

SPG stimulation produced complete abolition of pain within 3 min in 5 out of 15 headache peaks treated in 2 patients (33.3\%). In 3 of 15 attacks, $<50 \%$ headache relief was achieved. One patient had headache relief with SPG stimulation, but also with sham stimulation. No adverse effects occurred during this study. 
These results paved the way for the ongoing Pathway MIG-I trial, where the efficacy of effective stimulation with the ATI SPG Neurostimulator is being evaluated against sham stimulation for acute and preventive treatment in patients with frequent migraine. No data are available yet.

\section{Conclusions}

There is evidence that the SPG plays a role in the pathophysiology of neurovascular headaches. Based on available studies, the evidence looks much more convincing in $\mathrm{CH}$ than in migraine. In both headache types, however, there is a need for more efficient and better tolerable treatments, particularly in the most severely affected patients.

In experimental animals, low-frequency stimulation of the SPG produces vascular and meningeal changes that are thought to be associated with migraine and $\mathrm{CH}$. A contrario, stimulation at high frequency is thought to inhibit parasympathetic output by exhausting transmitter synthesis and release. Based on this rationale, proof-ofconcept studies have been performed with SPG stimulation by removable needle electrodes implanted in the pterygopalatine fossa during $\mathrm{CH}$ or migraine attacks, abolishing the pain in 61 and $33 \%$ of attacks, respectively.

The hitherto only sham-controlled RCT of SPG stimulation was performed in chronic $\mathrm{CH}$ with the implantable ATI Neurostimulator. In 14 out of 28 evaluable patients, effective SPG stimulation for 15 min produced significant pain relief in $61 \%$ of attacks, compared to $7 \%$ for ineffective stimulation. Incidentally, this study suggested that repeated SPG stimulation was also able to decrease attack frequency. Overall, 25\% of the patients had an acute response, 36\% had a frequency response, and $7 \%$ experienced both. The Pathway CH-I trial clearly shows that SPG stimulation with the implantable ATI Neurostimulator is able to abort $\mathrm{CH}$ attacks. The possible preventive effect needs to be verified in a proper RCT. The acute effect must be weighed against the adverse effects of the surgical intervention that are comprised of perioperative local symptoms and in particular long-lasting sensory disturbances due to injury of branches of the maxillary nerve.

The patients enrolled in the Pathway $\mathrm{CH}$-I trial were severely disabled chronic $\mathrm{CH}$ patients. These patients became resistant to the available preventive drugs, but their attacks usually still responded fully and rapidly (within 5-10 min) to injectable sumatriptan and sometimes to oxygen inhalation. Therefore, they are above all in need of a better preventive treatment. SPG stimulation was most effective in attacks of moderate severity and thus treated early. In severe attacks, e.g. which awake patients during night, SPG stimulation was much less effective than injectable sumatriptan. One major advantage of SPG stimulation, however, is that it can be repeated several times over a 24-hour period in patients with multiple attacks and can be used by patients with cardiovascular contraindications for injectable sumatriptan. 
Contrary to SPG stimulation, occipital nerve stimulation has a convincing preventive effect in refractory chronic $\mathrm{CH}$ patients as it is seldom useful during an attack. Therefore, the two neuromodulation methods are not comparable at this stage, and could be complementary. Assuming that the preventive effect of SPG stimulation can be proven in $\mathrm{CH}$, the effect size and, more importantly, the benefit/adverse effect ratio have to be compared with those of occipital nerve stimulation.

During migraine attacks, the outcome of the proof-of-concept study using a removable electrode implanted in the pterygopalatine fossa was less encouraging than in $\mathrm{CH}$ attacks since only 1 out of 3 attacks was interrupted. Nonetheless, an RCT of the effect of SPG stimulation with the ATI Neurostimulator (Pathway MIG-I) has been launched in frequent migraine. Besides a possible action on the attack, most patients and their treating practitioners would welcome a stimulation-induced reduction of attack frequency.

\section{References}

1 Sluder G: The role of the sphenopalatine (or Meckel's) ganglion in nasal headaches. NY Med J 1908;87: 989-990.

2 Headache Classification Committee of the International Headache Society (IHS). ICHD-III-beta: The International Classification of Headache Disorders, 3rd edition (beta version). Cephalalgia 2013;33:629808.

3 Devoghel JC: Cluster headache and sphenopalatine block. Acta Anaesthesiol Belg 1981;32:101-107.

4 Felisati G, Arnone F, Lozza P, Leone M, Curone M, Bussone G: Sphenopalatine endoscopic ganglion block: a revision of a traditional technique for cluster headache. Laryngoscope 2006;116:1447-1450.

5 Pipolo C, Bussone G, Leone M, Lozza P, Felisati G: Sphenopalatine endoscopic ganglion block in cluster headache: a reevaluation of the procedure after 5 years. Neurol Sci 2010;31(suppl 1):S197-S199.

6 Sanders M, Zuurmond WW: Efficacy of sphenopalatine ganglion blockade in 66 patients suffering from cluster headache: a 12- to 70-month follow-up evaluation. J Neurosurg 1997;87:876-880.

7 Narouze S, Kapural L, Casanova J, Mekhail N: Sphenopalatine ganglion radiofrequency ablation for the management of chronic cluster headache. Headache 2009;49:571-577.

8 Chua NH, Vissers KC, Wilder-Smith OH: Quantitative sensory testing may predict response to sphenopalatine ganglion pulsed radiofrequency treatment in cluster headaches: a case series. Pain Pract 2011; 11:439-445.

9 Meyer JS, Binns PM, Ericsson AD, Vulpe M: Sphenopalatine ganglionectomy for cluster headache. Arch Otolaryngol 1970;92:475-484.
10 Levin M: Nerve blocks in the treatment of headache. Neurotherapeutics 2010;7:197-203.

11 Khan S, Schoenen J, Ashina M: Sphenopalatine ganglion neuromodulation in migraine: what is the rationale? Cephalalgia 2014;34:382-391.

12 May A, Goadsby PJ: The trigeminovascular system in humans: pathophysiologic implications for primary headache syndromes of the neural influences on the cerebral circulation. J Cereb Blood Flow Metab 1999;19:115-127.

13 Goadsby PJ: Pathophysiology of cluster headache: a trigeminal autonomic cephalgia. Lancet Neurol 2002;1:251-257.

14 Jürgens TP, Schoenen J, Rostgaard J, Hillerup S, Láinez MJ, Assaf AT, May A, Jensen RH: Stimulation of the sphenopalatine ganglion in intractable cluster headache: expert consensus on patient selection and standards of care. Cephalalgia 2014;34:1100-1110.

15 Schytz HW, Barløse M, Guo S, Selb J, Caparso A, Jensen R, Ashina M: Experimental activation of the sphenopalatine ganglion provokes cluster-like attacks in humans. Cephalalgia 2013;33:831-841.

16 Akerman S, Holland PR, Lasalandra MP, Goadsby PJ: Oxygen inhibits neuronal activation in the trigeminocervical complex after stimulation of trigeminal autonomic reflex, but not during direct dural activation of trigeminal afferents. Headache 2009;49: 1131-1143.

17 Burstein R, Jakubowski M: Unitary hypothesis for multiple triggers of the pain and strain of migraine. J Comp Neurol 2005;493:9-14.

18 Maizels M, Geiger AM: Intranasal lidocaine for migraine: a randomized trial and open-label follow-up. Headache 1999;39:543-551. 
19 Piagkou M, Demesticha T, Troupis T, Vlasis K, Skandalakis P, Makri A, Mazarakis A, Lappas D, Piagkos G, Johnson EO: The pterygopalatine ganglion and its role in various pain syndromes: from anatomy to clinical practice. Pain Pract 2012;12:399-412.

20 The Sumatriptan Cluster Headache Study Group: Treatment of acute cluster headache with sumatriptan. N Engl J Med 1991;325:322-326.

21 Cohen AS, Burns B, Goadsby PJ: High-flow oxygen for treatment of cluster headache: a randomized trial. JAMA 2009;302:2451-2457.

22 Evers S, Afra J, Frese A, Goadsby PJ, Linde M, May A, Sandor PS: Cluster headache and other trigemino-autonomic cephalgias; in Gilhus NE, Barnes MP, Brainin M (eds): European Handbook of Neurological Management, ed 2. Oxford, Wiley-Blackwell, 2011, vol 1, pp 179-190.

23 Magis D, Schoenen J: Advances and challenges in neurostimulation for headaches. Lancet Neurol 2012;11:708-719.

24 Ansarinia M, Rezai A, Tepper SJ, Steiner CP, Stump J, Stanton-Hicks M, Machado A, Narouze S: Electrical stimulation of sphenopalatine ganglion for acute treatment of cluster headaches. Headache 2010;50: 1164-1174.
25 Schoenen J, Jensen RH, Lantéri-Minet M, Láinez MJ, Gaul C, Goodman AM, Caparso A, May A: Stimulation of the sphenopalatine ganglion (SPG) for cluster headache treatment. Pathway $\mathrm{CH}-1$ : a randomized, sham-controlled study. Cephalalgia 2013;33:816830.

26 Magis D, Bruno MA, Fumal A, Gérardy PY, Hustinx $\mathrm{R}$, Laurey S, Schoenen J: Central modulation in cluster headache patients treated with occipital nerve stimulation: an FDG-PET study. BMC Neurol 2011; 11:25.

27 Coppola G, Schoenen J: Management of acute and chronic migraine. Curr Opin Support Palliat Care 2012;6:177-182.

28 Silberstein SD, Latsko M, Schoenen J: Preventive antimigraine drugs; in Fernández-de-las-Peñas C Chaitow L, Schoenen J (eds): Contemporary Issues in Physical Therapy and Rehabilitation Medicine. Multidisciplinary Management of Migraine. Burlington, Jones \& Bartlett Learning, 2013, pp. 91-102.

29 Schoenen J, Vandersmissen B, Jeangette S, Herroelen L, Vandenheede M, Gérard P, Magis D: Migraine prevention with a supraorbital transcutaneous stimulator: a randomized controlled trial. Neurology 2013;80:697-704.

30 Tepper SJ, Rezai A, Narouze S, Steiner C, Mohajer P, Ansarinia M: Acute treatment of intractable migraine with sphenopalatine ganglion electrical stimulation. Headache 2009;49:983-989.
Prof. Jean Schoenen

Headache Research Unit, Department of Neurology

CHR Citadelle, Liège University

Blvd du XII de Ligne, BE-4000 Liège (Belgium)

E-Mail jschoenen@ulg.ac.be

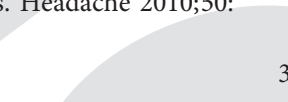

